\title{
Morbidity, mortality and long-term outcome of lung cancer resections performed in palliative intent
}

\author{
Emanuel Palade ${ }^{1,2}$, Jutta Günter ${ }^{1}$, Juan M. M. Gomez ${ }^{1}$, Ulrich F. Wellner ${ }^{2}$, Severin Schmid ${ }^{1,3}$, \\ Sebastian Wiesemann ${ }^{1,3}$, Bernward Passlick ${ }^{1,3}$ \\ ${ }^{1}$ Department of Thoracic Surgery, Medical Center - University of Freiburg, Freiburg im Breisgau, Germany; ${ }^{2}$ Department of Surgery, Medical \\ University of Schleswig-Holstein, Campus Lübeck, Lübeck, Germany; ${ }^{3}$ Faculty of Medicine, University of Freiburg, Freiburg im Breisgau, Germany \\ Contributions: (I) Conception and design: E Palade, B Passlick; (II) Administrative support: None; (III) Provision of study materials or patients: J \\ Günter, JM Gomez; (IV) Collection and assembly of data: E Palade, J Günter; (V) Data analysis and interpretation: E Palade, UF Wellner, B Passlick; \\ (VI) Manuscript writing: All authors; (VII) Final approval of manuscript: All authors. \\ Correspondence to: Emanuel Palade, MD. Ratzeburger Allee 160, 23538 Lübeck, Germany. Email: emanuel.palade@uksh.de.
}

Background: Surgery is seldom used for palliation in advanced lung cancer and the published data on this issue are very limited. We aimed to assess the results of palliative lung resections and identify criteria to guide surgical therapy in this situation.

Methods: This is a retrospective single-institution analysis of patients with palliative intended lung cancer resection. Survival analysis was performed by Kaplan-Meier method, log-rank test and Cox proportional hazards regression at a statistical significance level of $\mathrm{P}=0.05$.

Results: Forty-eight patients received palliative intended lung resections (31 lobectomies, 4 bilobectomies, 13 pneumonectomies) with acceptable rates of severe complications (Clavien-Dindo >IIIa 29\%) and 30-day mortality (4\%). The most frequent indications were infection and hemoptysis. The median survival for the entire group was 12 months (95\% CI: 6.9-17.1 months). Due to unexpectedly favorable histopathologic tumor stage, a switch to curative treatment in 17 completely resected patients resulted in a 2 -year survival rate of $46 \%$. In a subgroup of 20 patients with favorable prognostic factors as identified by uni- and multivariable analysis, a median survival of 26 months was observed.

Conclusions: In well selected patients with lung cancer, resection in palliative intent can offer symptom relief and even a survival benefit with acceptable morbidity and low mortality. Prognostic factors were identified and can be used to guide operative treatment. Due to the low specificity of CT and FDG-PET/ CT in the presence of inflammation or centrally located lung tumors, a large proportion of patients with lung resection in palliative intent experience a down-staging and frequently also a switch from palliative to curative treatment with additional survival benefit. Factors like expected complete resection, a squamous cell type and the ability to receive adjuvant therapy are useful to support the decision to perform palliative tumor resection.

Keywords: Palliative lung resection; advanced lung cancer; tumor necrosis; hemoptysis

Submitted Oct 17, 2018. Accepted for publication Aug 27, 2019.

doi: $10.21037 /$ jtd.2019.09.61

View this article at: http://dx.doi.org/10.21037/jtd.2019.09.61

\section{Introduction}

Worldwide, approximately 1.6 million new lung cancer cases are diagnosed and nearly 1.4 million lung cancer patients die every year. A large proportion of lung cancer patients need palliative care at some point in the course of disease (1). About $50 \%$ are already metastasized at the time of diagnosis and best supportive care (BSC) or a combination of BSC with chemotherapy is the standard of care for these patients (2).

The major goal of palliative care is to alleviate suffering 
by treating distressing symptoms. By combining early palliative interventions with oncologic therapy a significant improvement in outcome can be expected in patients with advanced non-small-cell lung cancer (NSCLC) (3). Further, the addition of chemotherapy to BSC results in significant improvement in overall-survival [hazard ratio (HR): 0.76; $95 \%$ confidence interval (95\% CI): $0.69-0.84 ; \mathrm{P}<0.001]$ as found by Zhong et al. in a meta-analysis of 16 randomized controlled trials including 4,135 patients. Although a significant relative risk (RR) reduction in 6-month (28\%, $\mathrm{P}=0.001), 12$-month $(11 \%, \mathrm{P}<0.001)$ and 2 -year mortality $(5 \%, \mathrm{P}=0.02)$ occurred, concerns remained regarding the higher toxicity of the combined therapy and the negative impact of the chemotherapy related morbidity on the quality of life of these fragile patients (2).

In patients with advanced lung cancer, various measures can be used to relieve a wide spectrum of symptoms. These methods can be divided into surgical and non-surgical. Surgical palliation includes treatment of pleural effusions, lung resections, management of airway obstruction and surgery for symptoms due to metastasis. Nonsurgical methods aim to control pain, cough, hemoptysis, respiratory insufficiency, fatigue, metabolic and humoral changes, anorexia, superior vena cava syndrome and other symptoms. Combinations of surgical and non-surgical methods are sometimes necessary. For the individual patient, the appropriate method has to be chosen according to performance status, life expectancy and the expected treatment result in terms of quality of life and survival.

Palliative lung resections are generally accepted but rarely performed operations. In this setting, the goal is to obtain an immediate and prolonged relief of the symptoms or to prevent severe local tumor complications without intention to cure $(4,5)$. Minimal requirements for surgery are the functional and technical resectability. As many candidates for palliative resections have an infected tumor or poststenotic pneumonia, the performance status is usually reduced. Severe comorbidities which can increase the operative risk and are not directly related to the tumor complication (e.g., sepsis by infection, anemia by bleeding) have to be taken in consideration and can preclude surgery. In contrast to curative intent resections, the tumor extension (N, M-status) as well as potentially positive resection margins $(\mathrm{R} 1, \mathrm{R} 2)$ are not essential parameters to influence the indication for surgery.

Typical indications for palliative lung resections are poststenotic pneumonia, tumor necrosis with abscess formation, life threatening hemoptysis or rarely intractable pain due to chest wall involvement. Other, less frequent indications are therapy refractory cough and central vascular occlusion of one lung (4-6).

The published experience with palliative lung resections is very limited and summarizes only a small number of case series. On the other hand, generating high quality evidence by well-designed comparative studies is not possible due to the rarity of this indication and the difficulties in creating a reference group as the indication for palliative resection implies that other methods are likely less effective.

Therefore, we analyzed our experience on palliative resections for lung cancer in an attempt to evaluate the potential benefits and to find objective criteria to support surgical decisions.

\section{Methods}

This work presents the results of a retrospective singleinstitution analysis of lung resections for lung cancer performed in palliative intent. Using the electronic patients' files, the study subjects were identified according to following search criteria: T3, T4, N2, N3, M1, R1, R2, poststenotic pneumonia, abscess, tumor necrosis, tumor infection and hemoptysis. The intent of the procedure (palliative or curative), was always formulated prior to resection and documented in the operation record by the surgeon in charge. This information is mandatory in our unit and serves to justify the procedure. The $7^{\text {th }}$ edition of the lung cancer classification and staging system was used.

Between 01/2008-12/2013, 703 patients with lung cancer were operated in our institution. For $n=48$ patients $(6.8 \%)$ a palliative intention was formulated prior to resection and were included in the analysis. Based on favorable changes in the tumor stage after resection compared with the clinical stage, for 17 patients with completely resected tumors a switch to curative therapy emerged. Two subgroups of patients resulted: "remained palliative" ( $\mathrm{n}=31)$ and "switched to curative" $(\mathrm{n}=17)$.

Treatment related outcomes (morbidity and mortality) and survival analysis (median survival, 95\% CI, 12-month survival rate, median follow-up time, Kaplan-Meier curves, log-rank P) were assessed at a statistical significance level of $\mathrm{P}=0.05$. Prognostic factors for survival and risk factors for operative morbidity and mortality were identified by univariable and multivariable analysis. The statistical analysis was performed using SPSS (version 22.0 for Windows, SPSS, Inc., Chicago, IL, USA). The study was approved by the Ethics committee of our institution (EKFreiburg 200/17). 
Table 1 Patients characteristics: age, sex, ASA-score, tumor location, histology and clinical tumor stage

\begin{tabular}{|c|c|}
\hline Parameter & Number \\
\hline Age (years) & Median 67, range $39-79$ \\
\hline \multicolumn{2}{|l|}{ Sex } \\
\hline Male & 42 \\
\hline Female & 6 \\
\hline \multicolumn{2}{|l|}{ ASA-score } \\
\hline ASA 3 & 24 \\
\hline ASA 4 & 24 \\
\hline \multicolumn{2}{|l|}{ Tumor location (right/left) } \\
\hline In one lobe & $19 / 19$ \\
\hline Centrally or lobe overlapping & $4 / 2$ \\
\hline Main bronchus & $4 / 0$ \\
\hline Total & $27(56 \%) / 21(44 \%)$ \\
\hline \multicolumn{2}{|l|}{ Histology } \\
\hline Squamous cell carcinoma & $27(56 \%)$ \\
\hline Adenocarcinoma & $13(27 \%)$ \\
\hline Large cell carcinoma & $5(11 \%)$ \\
\hline Sarcomatoid & $3(6 \%)$ \\
\hline \multicolumn{2}{|l|}{ Preoperative tumor stage } \\
\hline IIB & 1 \\
\hline $\mathrm{IIIA}_{3,4}(\mathrm{~T} 1-3 \mathrm{~N} 2)$ & 23 \\
\hline IIIA (T4N1) & 3 \\
\hline IIIB (T4N2) & 6 \\
\hline IIIB $\left(T_{n} N 3\right)$ & 2 \\
\hline IV M1a & 1 \\
\hline IV M1b & 12 \\
\hline
\end{tabular}

\section{Results}

The assessed baseline and treatment related characteristics for the 48 cases with palliative intended resection are listed in Tables 1 and 2.

The following symptoms and findings were indications for surgery: hemoptysis in $12(25 \%)$ cases, intractable infection (poststenotic pneumonia, tumor necrosis with abscess) in 40 (83\%), therapy refractory pain in 1 and other symptoms (1 tumor perforation with seropneumothorax, 1 empyema and 1 severe fatigue) in 3 . In 8 cases a combination of two symptoms (always infection associated in 6 with hemoptysis, in 1 with severe pain and in 1 with empyema) lead to lung resection. In 5 cases (10\%) the condition necessitating surgery (tumor abscess) emerged during chemotherapy.

The most frequent lung resection performed was lobectomy (65\%) followed by pneumonectomy (27\%). A total of 26 extensions of the resections were necessary in 17 patients (35.4\%): associated with lobectomy in 7 cases, bilobectomy in 2 and pneumonectomy in 8 .

In 30 patients $(62.5 \%)$ a total of 54 postoperative complications were encountered (Table 2). Regarding only the major complications (Clavien-Dindo $>\mathrm{III} a$ ) the incidence was much lower ( $\mathrm{n}=14,29 \%)(7)$. In 6 cases $(12.5 \%)$, a reoperation was necessary: one for persistent air leak, one for bleeding, two for bronchial stump insufficiency, one for destroyed lobe by necrotizing pneumonia after lobectomy and one for empyema. All of the 4 deaths recorded at 90 days after surgery were due to sepsis by pneumonia and none was tumor related.

At a median follow-up of 12.5 months (0-67 months) the median survival of the entire group was 12 months ( $95 \%$ CI: 6.9-17.1 months) (Figure 1).

Discrepancies between the preoperative and postoperative stage were common (Figure 2). For clarification, the very inhomogeneous stage IIIA was subdivided based on the different $\mathrm{T}$ and $\mathrm{N}$ stages. The resulting categories were ordered according to the expected survival in a decreasing sequence, using the HRs calculated by the International Association for the Study of Lung Cancer (8). Comparing the distribution of the stages (continuous and dotted lines in Figure 2) a shift from preoperatively higher stages to postoperative stages with better prognosis can be clearly observed.

Although all patients were considered initially incurable by surgical means the postoperative histopathological report revealed a more favorable situation (R0 resection, potentially curable TNM stage) in 17 (35.4\%) cases. Therefore, postoperatively taking this effect as selection criteria, two subgroups of patients resulted: one group of patients "remained palliative" while the second group of patients "switched to curative". The structure of the entire cohort and of the two subgroups resulting after resection is illustrated in Figure 3.

The median survival in the subgroup "switched to curative" was 20 months (95\% CI: 0-45.4 months), compared to 12 months (95\% CI: 6.5-17.4 months; $\mathrm{P}=0.176$ ) in the subgroup "remained palliative" (Figure 4). 
Table 2 Operation related parameters: indications, type of resections, type of extensions, resection margins, complications and mortality rates

\begin{tabular}{|c|c|}
\hline Parameter & Number \\
\hline \multicolumn{2}{|l|}{ Indication for surgery } \\
\hline Infection & 40 \\
\hline Hemoptysis & 12 \\
\hline Pain & 1 \\
\hline Other & 3 \\
\hline Total & 56 \\
\hline \multicolumn{2}{|l|}{ Type of resection } \\
\hline Lobectomy & $31(65 \%)$ \\
\hline Bilobectomy & $4(8 \%)$ \\
\hline Pneumonectomy & $13(27 \%)$ \\
\hline \multicolumn{2}{|l|}{ Type of extensions } \\
\hline Bronchial sleeve & 5 \\
\hline Angioplasty & 1 \\
\hline Intrapericardial resection & 11 \\
\hline $\begin{array}{l}\text { Pericardial resection with } \\
\text { reconstruction }\end{array}$ & 3 \\
\hline Atrial resection & 1 \\
\hline Tangential resection VCS & 1 \\
\hline Esophageal wall & 2 \\
\hline Segmentectomy & 2 \\
\hline Total & 26 \\
\hline \multicolumn{2}{|l|}{ Resection status } \\
\hline Ro & 38 (79\%) \\
\hline $\mathrm{R} 1$ & $8(17 \%)$ \\
\hline $\mathrm{R} 2$ & $2(4 \%)$ \\
\hline Operating time (median) & $181 \min (103-495 \mathrm{~min})$ \\
\hline \multicolumn{2}{|l|}{ Postoperative tumor stage } \\
\hline IB (T2aN0) & 2 \\
\hline IIA (T2bN0) & 1 \\
\hline IIB (T3N0) & 4 \\
\hline IIIA (T3N1) & 11 \\
\hline IIIA (T1-3N2) & 12 \\
\hline IIIA (T4N0) & 1 \\
\hline IIIA (T4N1) & 2 \\
\hline IIIB (T4N2) & 2 \\
\hline $\mathrm{IIIB}\left(\mathrm{T}_{\mathrm{n}} \mathrm{N} 3\right)$ & 1 \\
\hline IV M1a & 1 \\
\hline IV M1b & 11 \\
\hline
\end{tabular}

Table 2 (continued)
Table 2 (continued)

\begin{tabular}{lc}
\hline Parameter & Number \\
\hline Complications & 13 \\
Pneumonia & 7 \\
Mechanical ventilation & 4 \\
Sepsis, MOF & 9 \\
Atrial fibrillation & 1 \\
Myocardial infarction & 2 \\
Acute renal failure & 1 \\
Bleeding & 2 \\
Stump insufficiency & 2 \\
Empyema & 2 \\
Recurrent nerve injuries & 3 \\
Persistent air leak (>7 days) & 1 \\
Pneumothorax & 5 \\
Wound infection & 2 \\
Postoperative psychosis & 54 \\
Total & 2
\end{tabular}

Patients necessitating transfusion 9 patients, 21 blood units

30-day mortality $2(4 \%)$

90-day mortality $4(8 \%)$

MOF, multiple organ failure.

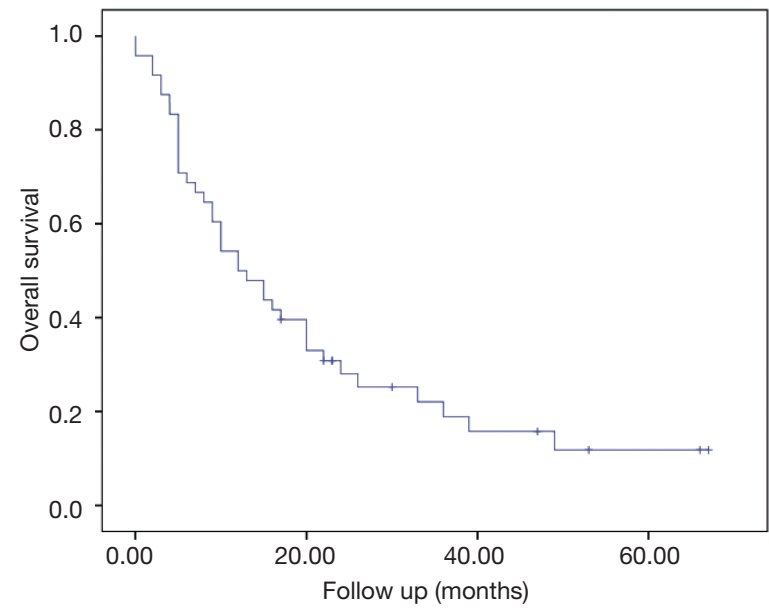

Figure 1 Overall survival of the entire group (Kaplan-Meier).

The 1-, 2- and 5-year survival rates for the entire cohort and the two resulted subgroups are presented in Table 3.

The univariable survival analysis included all assessed clinical parameters (Table 4). In multivariable analysis, 


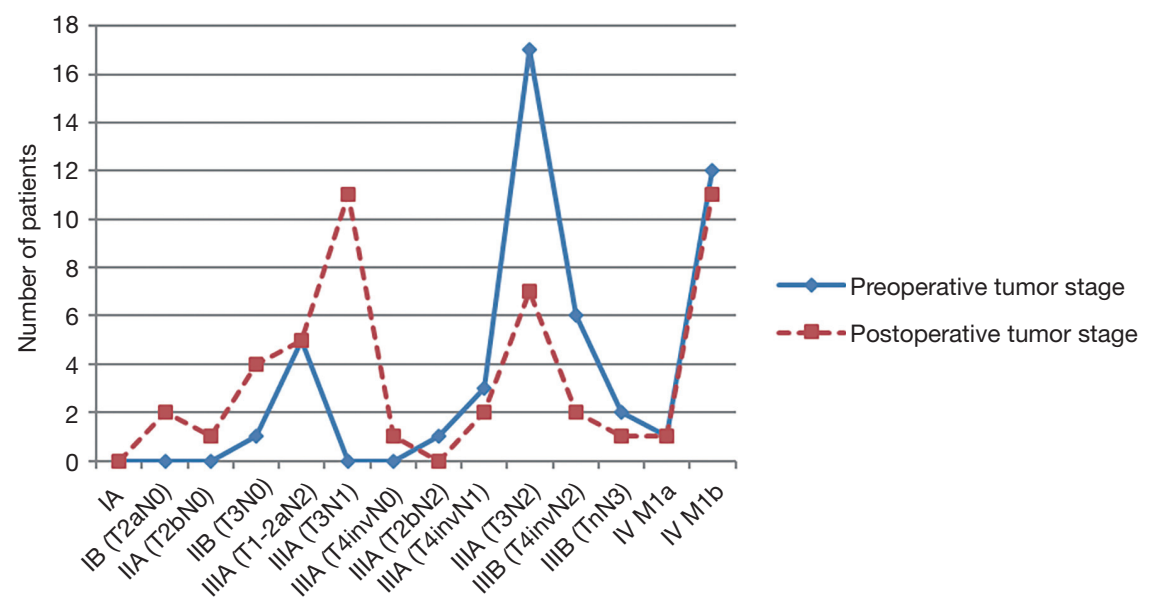

Figure 2 Comparative illustration of the preoperative and postoperative tumor stage.

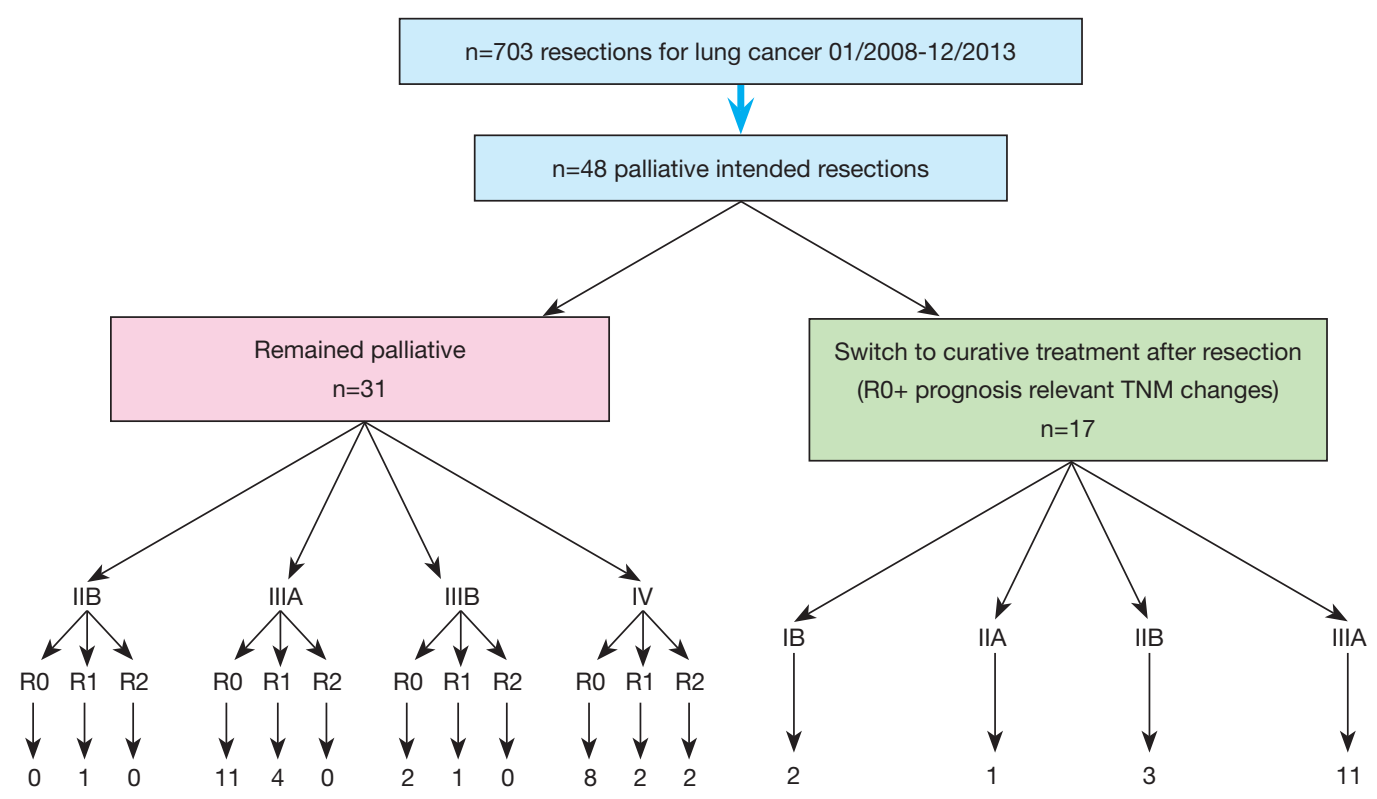

Figure 3 Diagram presenting the entire cohort and the two subgroups ("remained palliative", "switched to curative") resulted after resection. Due to multilevel or bulky N2 some IIIA and IIIB patients remained palliative despite R0 resection status.

three parameters were identified as independent prognostic factors: resection margin status $(\mathrm{P}=0.003)$, morbidity $(\mathrm{P}=0.017)$ and $\mathrm{T}$ stage $(\mathrm{P}=0.027)$. For the adjuvant therapy $(\mathrm{P}=0.051)$ and histologic type $(\mathrm{P}=0.061)$, a trend towards significance resulted (Tables 4 and 5 ).

Using the results of multivariable analysis and based on a clinical decision perspective, a subgroup of 20 patients ( $42 \%$ ) with favorable outcome was identified ("best group”). All patients had a locally completely resected (R0) squamous cell carcinoma and received adjuvant therapy. As in the setting of palliative surgery technical resectability precludes over locally tumor extent (T stage) and morbidity occurs first after operation and cannot be reliably predicted preoperative, these two parameters ( $\mathrm{T}$ stage and operative morbidity) although significant as prognostic factors, were not taking in regard for defining this subgroup.

A median survival of 26 months (95\% CI: 7.5-44.5 months) was observed in this subgroup. In those cases of the "best 


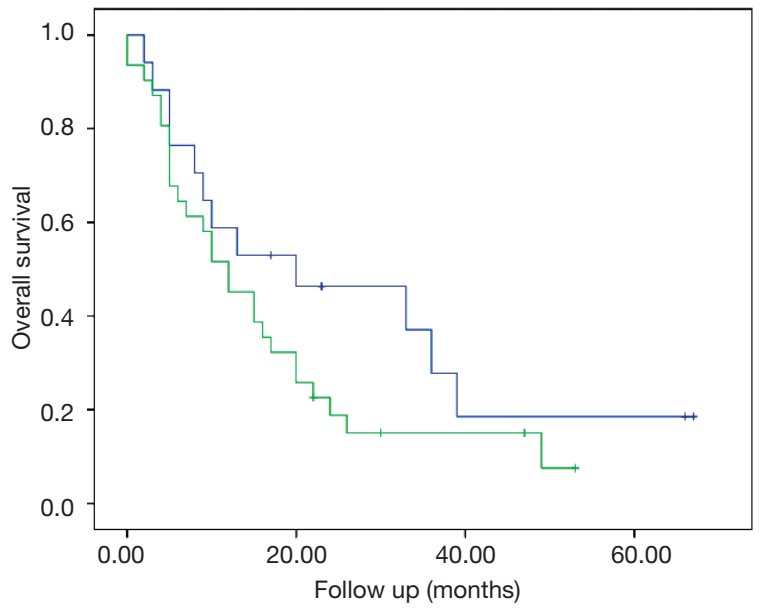

Figure 4 Comparative survival of the two postoperative resulted subgroups (blue line "switched to curative" $v s$. green line "remained palliative”) (Kaplan-Meier, $\mathrm{P}=0.176)$. group" with additional postoperative conversion to curative treatment ( $\mathrm{n}=9,19 \%)$, the median survival was even much better (median 36 months, 95\% CI: 0-81.5 months).

\section{Discussion}

Despite substantial achievements in diagnostic and therapy of lung cancer, a large number of patients worldwide experience debilitating symptoms due to advanced tumor urging the need of palliative care. Although lung resections in palliative intention represent a generally accepted treatment modality, this kind of surgery is rarely performed and the published experience on this topic is very limited (4-6). Therefore, conclusive evidence regarding outcome and objective selection criteria are lacking.

Some aspects are essential in decision-making for

Table 3 Survival rates of the entire cohort and of the two postoperative resulted subgroups

\begin{tabular}{lccc}
\hline Survival & Entire cohort (\%) & Subgroup "curative" $(\mathrm{n}=17)(\%)$ & Subgroup "palliative" $(\mathrm{n}=31)(\%)$ \\
\hline 1-year & 50 & 59 & 45 \\
2-year & 28 & 46 & 19 \\
5 -year & 12 & 19 & - \\
\hline
\end{tabular}

Table 4 Univariable survival analysis. P values derived from two-sided Chi squared and log-rank tests

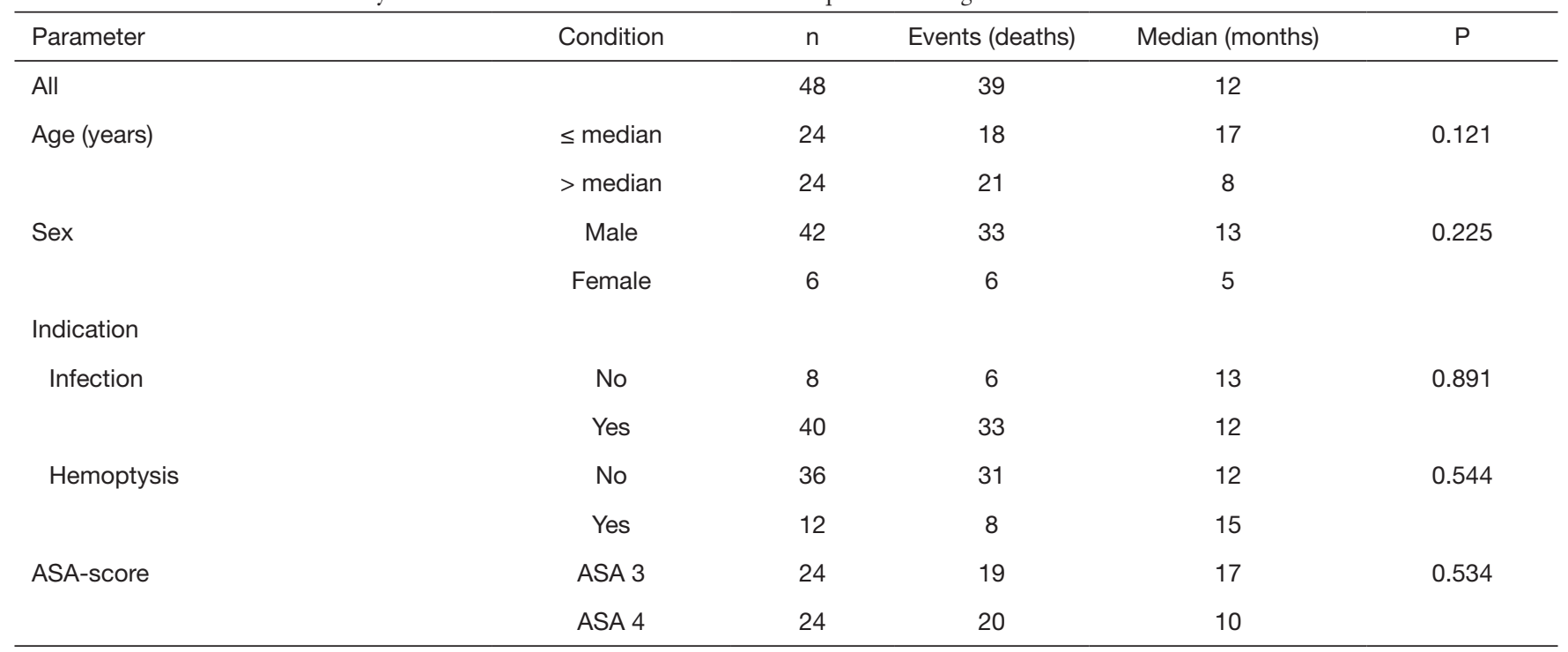

Table 4 (continued) 
Table 4 (continued)

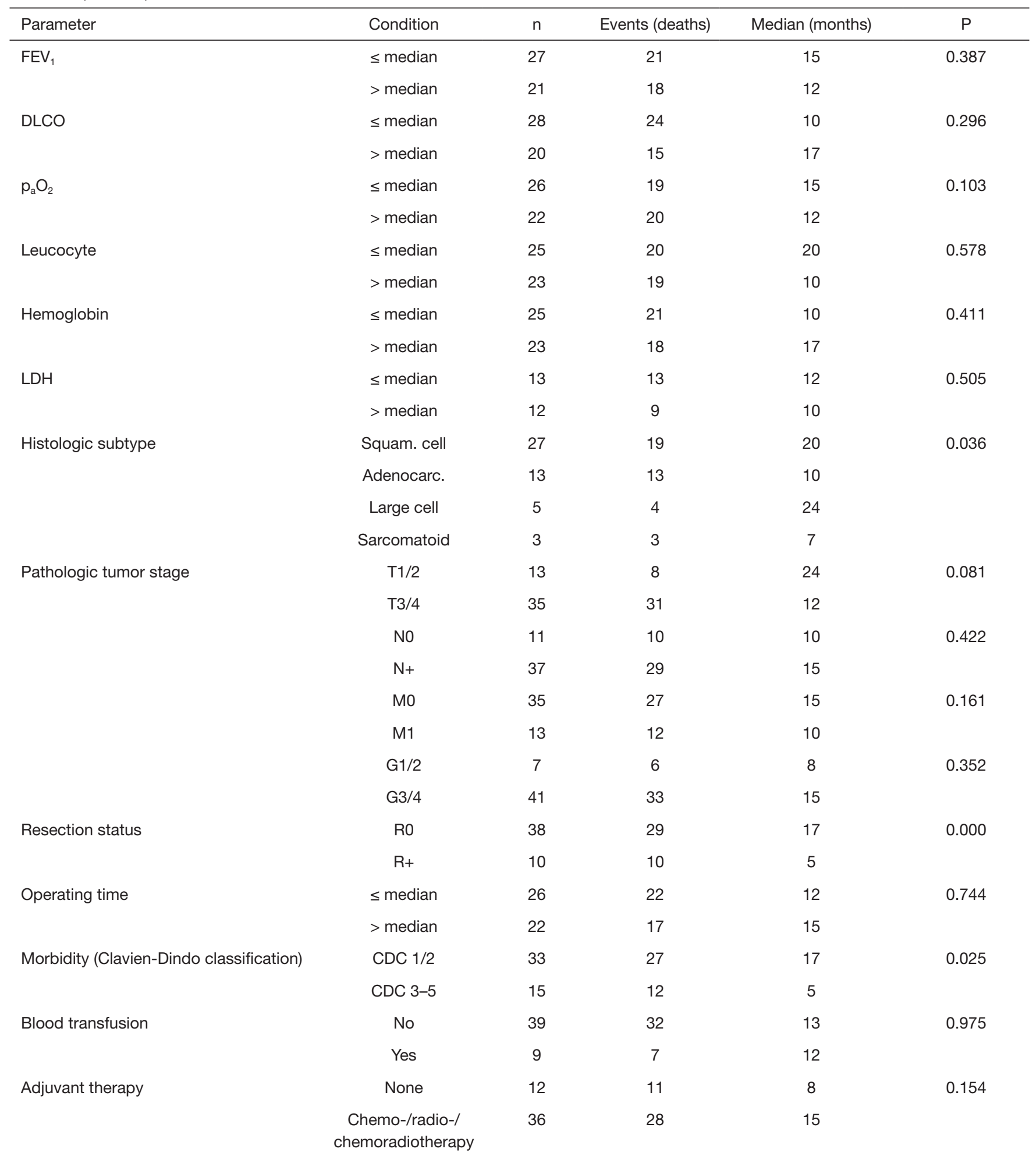

FEV1, forced expiratory volume in the first second; DLCO, diffusion capacity for carbon monoxide; $\mathrm{p}_{\mathrm{a}} \mathrm{O}_{2}$, arterial partial pressure of oxygen; LDH, lactate dehydrogenase. 
Table 5 Multivariable survival analysis identifying significance or a trend towards significance for resection margin status, operative morbidity (Clavien-Dindo classification), T stage, adjuvant therapy and histologic type. P values derived from two-sided Chi squared and Cox-regression tests

\begin{tabular}{lcc}
\hline Parameter & Hazard ratio & $95 \% \mathrm{Cl}$ \\
\hline Resection margin (R+ vs. R0) & 3.57 & $1.55-8.25$ \\
Operative morbidity (Clavien-Dindo 3-5 vs. Clavien-Dindo 0-2) & 2.49 & $1.17-5.28$ \\
T stage (T3/4 vs. T1/2) & 2.51 & $1.11-5.68$ \\
Adjuvant therapy (yes vs. no) & 0.47 & 0.003 \\
Histologic type (squamous cell carcinoma vs. all other) & 0.63 & 0.017 \\
\hline
\end{tabular}

palliative lung surgery. From the surgical point of view, the resection must be technically feasible and should address severe or potentially life threatening symptoms. Furthermore, surgery should be considered only when no other less invasive methods can be offered with potentially similar results in terms of symptom control and life expectancy.

The classical indications are: hemoptysis, poststenotic pneumonia with abscess formation, tumor necrosis with fatigue, centrally vascular occlusion, recurrent pleural effusion, intractable cough or pain (4). In our cohort, the main indication was infection $(40 \%)$ followed by hemoptysis $(12 \%)$ with a combination of two symptoms in 8 cases. In 5 cases $(10 \%)$ the situation necessitating surgery (tumor abscess) emerged under chemotherapy. Other indications were very rare or not encountered in our group, as currently such symptoms are managed mostly by nonsurgical methods.

Due to the high number of locally advanced tumors $(\mathrm{n}=19,40 \%)$, the incidence of pneumonectomies $(\mathrm{n}=13$, $27 \%$ ), the rate of extended resections (35\%) and the rate of incomplete resections $(21 \%)$ were increased. Interestingly, no intraoperative adverse events were encountered and the need for blood transfusion was low (21 blood units in 9 patients). The duration of the operations was in median only about 30 min longer as for open resections in patients with early stage lung cancer as compared with recently published data from our institution (181 vs. $158 \mathrm{~min}$ ) (9). For curative intent operations, as found by large database analysis such as those from the Society of Thoracic Surgeons (9,033 pulmonary resections performed 1999 to 2006) or from the Commission on Cancer of the American College of Surgeons (11,668 patients in 729 hospitals in $2001)$, these rates are considerably lower $(10,11)$. In these studies, the pneumonectomy rate is $6.5 \%$ respectively $13.6 \%$, the incidence of positive resection margins is $7.8 \%$. A blood transfusion was used in $7 \%$ of the patients.

\section{Operative morbidity and mortality}

Compared with postoperative data regarding lung resections in curative intent, the overall postoperative morbidity in our group was considerably higher. This parameter lies almost two-fold higher than in the STS registry-based cohort (62.5\% vs. $32 \%)$. Referring only to the major complications in our group (Clavien-Dindo $>\mathrm{III}$ ), this proportion is much lower (29\%) and allows the assumption that palliative resections can be performed with acceptable morbidity (7).

Despite the much higher rate of pneumonectomies and the high ASA-score (American Society of Anesthesiologists) of our patients, the 30 -day mortality (4\%) was similar to the incidence published by the STS (2.5\% operative mortality) and CCACS study (3.2\% for high-volume centers as ours). Furthermore, in the last study the operative mortality after pneumonectomy reached $8.5 \%$.

A meaningful comparison of our data with results from other studies on palliative intent lung resections is nearly impossible due to the scarcity of publications on this issue. Liewald et al. [2001] analyzed a total of 75 patients categorized into 3 groups: palliative intent with complete resection ( $\mathrm{n}=11)$, palliative intent and incomplete resection $(n=23)$ and curative intent with incomplete resection $(\mathrm{n}=38)$. The incidence of pneumonectomies $(56 \%)$ and extended resections $(62.6 \%)$ were even higher than in our group. The same holds true for the incidence of margin positive resection $(68 \%)$ and 30 -day mortality (12\%) of palliative intent operations. In palliative resections, a clear survival benefit was observed for the patients in which a complete resection was achieved (median overall survival 25.5 vs. 7.7 months. The authors also demonstrate that frequently (32\% of cases), the initial palliative intent can turn into a curative therapy after a complete resection, 
and that in cases with severe tumor infection a sometimes spectacular improvement of the symptoms occurs even after pneumonectomy (4).

\section{Analysis of survival}

In a recently published meta-analysis of 16 randomizedcontrolled trials comparing chemotherapy plus BSC versus BSC alone in patients with non-small cell lung cancer, an significant advantage in overall survival occurred for the combination chemotherapy plus BSC (HR $=0.76$; 95\% CI: $0.69-0.84 ; \mathrm{P}<0.001)$, as well as a $\mathrm{RR}$ reduction of $28 \%$ for 6-month mortality (95\% CI: $12-40 \% ; \mathrm{P}=0.001$ ), $11 \%$ at 12 -month (95\% CI: $8-15 \% ; \mathrm{P}<0.001)$ and $5 \%$ at $24-$ month (95\% CI: $1-8 \%$; $\mathrm{P}=0.02$ ) (2). Regarding the original data from these 16 trials, in most studies, the median survival ranged between 3.8 and 5.9 months for BSC and 5 to 8.5 months for chemotherapy plus BSC. Furthermore, 12 -months survival rates ranged between $8 \%$ and $23 \%$ for BSC and $21 \%$ to $32 \%$ for chemotherapy plus BSC (2).

In our cohort, the median survival for both the entire group and the subgroup "remained palliative" was 12 months. This is in our opinion highly relevant as all our patients had severe tumor complications and an ASAcategory of 3 or 4 . The same was observed for 12-month survival (entire cohort $50 \%$, subgroup "switched to curative" 59\%, subgroup "remained palliative" 45\%). Although a direct comparison with data emerged from studies on conservative palliative treatment is not possible, it seems to be justified to offer palliative resections whenever feasible.

An interesting observation in our cohort was that in 17 cases (35\%), due to postoperative downwards changes in the clinically presumed tumor extent and in association with an achieved complete resection, a curative treatment resulted. The main reason is the low specificity of CT and FDGPET/CT (FDG-PET/CT = fluorodeoxyglucose positron emission tomography and computed tomography scan) in cases of inflammation and/or centrally located tumors. In a recent study by Kaseda et al., the rate of false-positive hilar and mediastinal lymph nodes in 388 operated lung cancer patients clinical staged I-IIIA non-small cell lung cancer was $43.8 \%$. Two risk factors for false-positive FDG-uptake were found: a history of lung disease (e.g., infection, inflammatory disease) and the central location of the tumor (12). In our setting of palliative resections, the decision to operate is independent from nodal status and therefore invasive mediastinal staging is not indicated.
The difference in median survival between the subgroups "switched to curative" and "remained palliative" was not significant (20 vs. 12 months, $\mathrm{P}=0.176$ ), probably due to the small size of the groups (17vs. 31).

\section{Criteria to support palliative intent lung resections}

As already mentioned and based on the acceptable morbidity (Clavien-Dindo >IIIa 29\%) and low mortality (4\%) after palliative resections for lung cancer, these resections can bring a survival benefit for patients with severe tumor related complications. Further, two additional reasons were identified and should justify the indication to palliative intent lung cancer resection: the high proportion of cases with good prognosis ("best group", 42\%) and the switch to curative treatment in a large proportion of patients (35\%).

Based on the uni- and multivariable analysis, three independent prognostic factors (resection status, postoperative morbidity and $\mathrm{T}$ stage) were identified. For two more factors (adjuvant therapy and histologic type), a trend towards significance resulted. These findings are not surprising and represent already accepted prognostic factors for curative resections in lung cancer. Maintaining the palliative intention of the therapy, a subgroup of 20 patients (locally completely resected squamous cell carcinomas, adjuvant therapy) was identified. The local extent of the tumor ( $\mathrm{T}$ stage) and postoperative morbidity were not considered, as these parameters did not influence the decision to operate. In this subgroup ("best group") a median survival of 26 months (95\% CI: 7.5-44.5 months) was observed, being similar to the median survival (1.9 years) reported by Ploeg et al. for operated stage II non-small cell lung cancer (13).

\section{Limitations of the study}

The limitations of our study are the retrospective nature and the analysis of a relatively small but highly selected group of patients with a great heterogeneity in tumor stage. Further, various pre- and postoperative therapies with impact on postoperative course and survival were performed.

As the published experience with palliative resections is very limited and generating evidence from well-designed comparison studies is not possible due to the rarity of this indication, the interpretation and comparison of the results can only be made on the basis of historical data on patients with advanced lung cancer. 
The important aspects of our study are the analysis of stage migration after resection, underlining the limitations of thoracic imaging (CT and FDG-PET/CT) in cases with centrally located tumors or inflammation and second, the identification of selection criteria to support decision making for surgery.

\section{Conclusions}

In our opinion, in well selected cases with technically resectable advanced lung cancer, palliative resections are indicated for severe complications like infection and/or hemoptysis. The decision must take into account alternative methods, potential risks and presumed life expectancy. For the operated cases, a survival benefit can be expected with acceptable morbidity and relatively low mortality, especially considering that in palliative resections, high rates of pneumonectomy, extended resections and patients with high ASA-score are encountered. In a significant proportion of cases, a down-staging may be observed after surgery compared to the clinical stage assessed by CT or PET/CTscan and a switch to curative treatment may result.

Identification of factors like expected complete resection, a squamous cell type and the ability to receive adjuvant therapy are arguments which might favor tumor resection. These favorable factors can be found in a large proportion of cases (about $42 \%$ ) and were associated with a median survival up to 26 months in our study.

These results motivate us to encourage chest physicians to interdisciplinary assess palliative lung cancer patients with experienced thoracic surgeons, and the later to publish their experience in an attempt to better clarify the role of surgery in this restricted group of patients with advanced lung cancer.

\section{Acknowledgments}

None.

\section{Footnote}

Conflicts of Interest: The authors have no conflicts of interest to declare.

Ethical Statement: The authors are accountable for all aspects of the work in ensuring that questions related to the accuracy or integrity of any part of the work are appropriately investigated and resolved. The study was approved by the Ethics committee of our institution (EKFreiburg 200/17).

\section{References}

1. Simone CB 2nd, Jones JA. Palliative care for patients with locally advanced and metastatic non-small cell lung cancer. Ann Palliat Med 2013;2:178-88.

2. Zhong $\mathrm{C}$, Liu H, Jiang L, et al. Chemotherapy plus best supportive care versus best supportive care in patients with non-small cell lung cancer: a meta-analysis of randomized controlled trials. PLoS One 2013;8:e58466.

3. Otsuka M, Koyama A, Matsuoka H, et al. Early palliative intervention for patients with advanced cancer. Jpn J Clin Oncol 2013;43:788-94.

4. Liewald F, Förster R, Halter G, et al. Chances in surgical therapy of lung carcinoma under palliative condition. Zentralbl Chir 2001;126:217-22.

5. Surgical management of non-small cell lung cancer. In: Pearson's thoracic and esophageal surgery; 3rd Edition, Elsevier 2008:799.

6. Kvale PA, Selecky PA, Prakash UB, et al. Palliative care in lung cancer: ACCP evidence-based clinical practice guidelines (2nd edition). Chest 2007;132:368S-403S.

7. Dindo D, Demartines N, Clavien PA. Classification of surgical complications: a new proposal with evaluation in a cohort of 6336 patients and results of a survey. Ann Surg 2004;240:205-13.

8. Goldstraw P, Crowley J, Chansky K, et al. The IASLC Lung Cancer Staging Project: proposals for the revision of the TNM stage groupings in the forthcoming (seventh) edition of the TNM Classification of malignant tumours. J Thorac Oncol 2007;2:706-14.

9. Palade E, Passlick B, Osei-Agyemang T, et al. Videoassisted vs open mediastinal lymphadenectomy for Stage I non-small-cell lung cancer: results of a prospective randomized trial. Eur J Cardiothorac Surg 2013;44:244-9; discussion 249.

10. Boffa DJ, Allen MS, Grab JD, et al. Data from The Society of Thoracic Surgeons General Thoracic Surgery database: the surgical management of primary lung tumors. J Thorac Cardiovasc Surg 2008;135:247-54.

11. Little AG, Rusch VW, Bonner JA, et al. Patterns of surgical care of lung cancer patients. Ann Thorac Surg 2005;80:2051-6; discussion 2056.

12. Kaseda K, Watanabe K, Asakura K, et al. Identification of false-negative and false-positive diagnoses of lymph node metastases in non-small cell lung cancer patients staged 
by integrated (18F-)fluorodeoxyglucose-positron emission tomography/computed tomography: A retrospective cohort study. Thorac Cancer 2016;7:473-80.

13. Ploeg AJ, Kappetein AP, van Tongeren RB, et al. Factors associated with perioperative complications and long-term results after pulmonary resection for primary carcinoma of the lung. Eur J Cardiothorac Surg 2003;23:26-9.

Cite this article as: Palade E, Günter J, Gomez JM, Wellner UF, Schmid S, Wiesemann S, Passlick B. Morbidity, mortality and long-term outcome of lung cancer resections performed in palliative intent. J Thorac Dis 2019;11(10):4308-4318. doi: $10.21037 /$ jtd.2019.09.61 